\title{
Design and Optimize FQPSK Modulation Concatenated with LDPC Code for Space Information Network
}

\author{
Zhi-dong Xie ${ }^{1, a^{*}}$, Chao $\mathrm{He}^{1,2, \mathrm{~b}}$, Jing $\mathrm{Hu}^{1, \mathrm{c}}$ and Dong-ming Bian ${ }^{1, \mathrm{~d}}$ \\ ${ }^{1}$ College of Communication Engineering,PLA University of Science and Technology, Nanjing, \\ China \\ 2 Engineer Academy of PLA, Xuzhou, China \\ axzd313@163.com, ${ }^{b}$ hechao619@163.com, xiaoxiaomissy@hotmail.com, dbdm@163.com
}

\begin{abstract}
Keywords: FQPSK; LDPC; coded modulation; space information network
Abstract. Because of the limited power and long propagation distance between spacecraft and Earth station, the high power efficiency is more important for space information system than others terrestrial communication system. Motivated by the potentially large gain achievable with iterative decoding, one novel coded modulation scheme based on LDPC code and FQPSK (Feher's QPSK) is designed and optimized in this paper, which can alleviate the challenge of signal power huge loss of communications path in space information system. The novel scheme improves power efficiency utilizing the property that LDPC code enables communication at very low signal noise rate and constant envelope property of FQPSK enables high power amplifier work at saturation states without nonlinear impact. At receiver, using iterative receive algorithm, large gain can be attained. The paper proposes concatenation scheme and optimizes it by computer assisted simulation. At last, the bit error rate performance of optimal scheme is confirmed.
\end{abstract}

\section{Introduction}

Long distance transmission is one of most rigorous difficulty in space information network compared with terrestrial communications system, which would lead to large transmission delay and huge signal power loss. For issues of the delay, which is an objective existence, it can be resort to networking, routing and protocol designing and so on. However, due to the long distance and unique operation environment, huge free space loss and large transmission delay in them are more significant than others to design a communications system between spacecraft and Earth station. The huge signal power loss could be resolved by increase of the transmission power and gain of antenna, reducing the noise temperature of receiver, adopting high efficient channel code and modulation, and so on. It has been a challenging issue how to meet the requirement for receiving very weak signal which induced by huge power transmission loss in space communications. Generally, modulation and channel coding both are effective methods to resolve the problem of power limited [1].

Channel coding is one of the efficient methods to increase power efficiency of the system so that almost all of space craft launched since 1960s adopted channel coding without any exception. Low-density parity-check (LDPC) codes has been attracted much attention due to its approaching channel capacity limit performance and feasible complexity during past years [2]. J. Thorpe and etc.'s contributions are most representative one, and Accumulate Repeat-4 Jagged Accumulate (AR4JA) code [3] proposed by them has been selected as recommendation by Consultative Committee for Space Data System (CCSDS) at 2007 year.

The space aircraft generally uses nonlinear high power amplifier (HPA) in order to fully utilize the power resource, and the HPA usually works at the saturation state to get the highest power efficiency. It leads to the fact that the channel of space communication is nonlinear [1]. Therefore, it is urgently required that the constant or quasi constant envelope modulation method would be employed in space communications to reduce the impact due to the nonlinearity of HPA. Feher Quadrature Phase Shift Keying (FQPSK) [4] [5] was invented by K. Feher, which is a quasi-constant envelope modulation with excellent spectral and power performance and can be used for space communication. Constant 
Envelope Enhanced FQPSK (CEEFQPSK), we proposed in [6], is an improved modulation based FQPSK with better power and spectral performance.

However, there is a non-neglectable performance deteriorating ascribed to independent design modulation and channel coding in traditional system. Such as in CCSDS protocol stack, modulation belongs to Physical Layer while channel coding belongs to Link Layer, which leads to convenience for designing and standardization, but face a notable performance loss.

Motivated by the potentially large coding gain achievable with iterative receiving using a soft input soft output (SISO) a posteriori probability (APP) algorithm [7], much attention has been paid for coded modulation concatenation. LDPC code concatenating modulation is studied widely [8] [11], major in concatenation with high order modulation and optimization for code designing. Demodulation of FQPSK also is a research hot spot during past years. However, little stress has been put on coded FQPSK except paper [12] [14]. Paper [12] gave the performance of concatenation of convolutional code and FQPSK using iterative decoding and demodulation. Unfortunately, there is any specific decoding algorithm in the paper. Paper [13] investigated the performance coded FQPSK in which it is presented as continuous phase modulation (CPM) by Laurent decomposition. Due to the fact that FQPSK can't be decomposed into closed form, the results only are approximative.

In this paper, we consider serially concatenated schemes with outer AR4JA codes and inner FQPSK modulations. The concatenation scheme enables the HPA to work at saturated state without need to back off owning to constant envelope of modulation signal. Large coding gain is obtained from AR4JA. At the same time, additional gain can be achieved by iterative decoding and demodulation for concatenation.

The paper is arranged as follows: the concatenation scheme is given in section II, including the system model and iterative receiving algorithm. The simulation and optimization is illustrated in section III and section IV concludes the paper.

\section{Concatenation Scheme}

\section{System model}

The transmitter in the concatenation scheme, shown in Fig. 1, consists of a simple concatenation of an outer AR4JA encoder and an inner FQPSK modulator. The binary bit streams $\left\{x_{i}\right\}$ are encoded by AR4JA encoder firstly. Then, the encoded data sequence $\left\{c_{k}\right\}$ are modulated by FQPSK modulator and transmitted. One interleaver can be employed in system between AR4JA encoder and FQPSK modulator or not according to the requirement of system design and performance. It can be implemented expediently due to the fact that it does not increase the complexity of signal processing procedure. In the system, the FQPSK modulator can be referred to [4] or can be CEEFQPSK proposed in [6] too, which is a strictly constant envelope and with better BER performance than FQPSK. The AR4JA encoder would be code encoder proposed in [3], which is a quasi-cyclic systemic code and can be realized by simple shift registers with linear complexity. The choice of modulation and AR4JA code both are compatible with CCSDS recommendations, which aim for generalizing in future space communications.

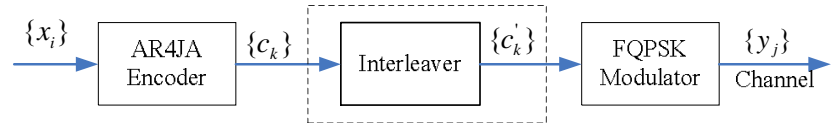

Fig.1 Sender of system

The receiver adopted in system is partitioned into two blocks, denoted as block A and block B, depicted in Fig. 2. At the input of the receiver, the sequence of channel observations is denoted as $\left\{r_{k}\right\}$. Iterative decoding and demodulation are achieved by message passing between Block A and Block B. Block A comprises the following sub-blocks.

ü A FQPSK SISO block matched to the FQPSK and the channel. This block computes the a posteriori reliabilities of the binary symbols $\left\{c_{k}\right\}$ on the basis of the channel observations and 
the relevant a priori reliabilities (coming from the block labeled "AR4JA VND" and described below) using APP SISO algorithm.

ü An AR4JA variable node detector (VND). This block computes the reliability of each binary symbol based on the reliabilities from the FQPSK SISO block and the information received from block and based on the code constraints.

Block B includes the AR4JA check node detector (CND). The AR4JA CND computes the reliability of each binary symbol $\left\{c_{k}\right\}$ based on the a priori reliabilities received from the AR4JA VND and based on the AR4JA code constraints.

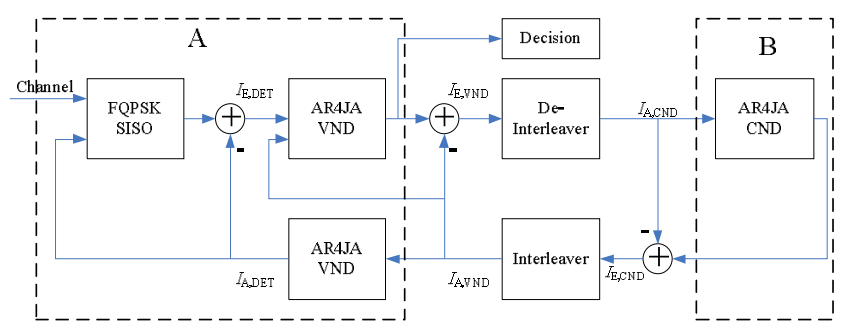

Fig.2 Iterative receiver of system

\section{Iterative Receiving Algorithm}

Using modified SISO APP algorithm for FQPSK presented in paper [14], the overall iterative receiving algorithm at the receiver can be described as follows.

1) As initialization step, the priori reliabilities of the symbols $\left\{c_{k}\right\}$ at the input of block A (from block B) correspond to complete uncertainty, which equal to 0 in the LL domain.

2) Block A computes output reliabilities and sends them to block B.

3) The AR4JA CND in block B computes the extrinsic information to be passed to block A.

4) The algorithm iterates from the step 2) until a valid AR4JA code is obtained or a maximum number of iteration has been achieved.

5) In the case a valid AR4JA code is not obtained, an additional standard AR4JA decoding algorithm is applied based on the last extrinsic information at the input of AR4JA VND block. This corresponds to iterating information only between AR4JA VND and AR4JA CND. The maximum number of standard LDPC decoding iterations is $\mathrm{N}$.

6) At the end of the process, the complete (not extrinsic) reliabilities are computed by the AR4JA VND and delivered to the decision.

In the algorithm above, The AR4JA CND in Block B computes the reliability of each binary symbol based on the a priori reliabilities received from the AR4JA VND and based on the AR4JA code constraints.

The reliabilities at the output of block A are computed as follows.

1) The VND processes the messages coming from block $B$ at each variable node by performing a sum of all the incoming messages excluding the one coming from the FQPSK-SISO block. The obtained messages are passed to the FQPSK-SISO block as a priori input.

2) The FQPSK-SISO block computes reliability values according to its internal algorithm based on the observations from the channel and the a priori information.

Finally, the VND computes the messages according to the standard LDPC decoding algorithm usingthe messages from the FQPSK-SISO decoder as a priori input.

\section{Performance Simulation and Optimization}

The FQPSK can be concatenated with AR4JA code when a SISO APP algorithm is adopted. However, there is no any reference for the performance of this scheme. In order to verify the performance of it, several computer simulations have been carried out. In the simulation, the (2048, 1024) AR4JA code is adopted, the maximum iteration times is 30 and AWGN channel is assumed. At last, an optical concatenated scheme has been proposed. 


\section{Optimization for Concatenation Scheme.}

According to the prevenient outcome, the trellis structure of FQPSK and interleaver has a significant effect on performance of the scheme. Therefore, it is need to optimize the concatenation scheme to get better performance.

In order to get a coding gain resulting from the interleaving process between the inner and outer codes, all component code should be recursive in parallel concatenation and inner code should be recursive at least in serial concatenation. Due to the fact that FQPSK can only be used as inner code, it must be with recursive structure when it is concatenated to convolutional code [10]. We have done simulation for the concatenation scheme with FQPSK and AR4JA code, both for non-recursive FQPSK and recursive one, with iteration times for decoding is 10. The influence of FQPSK trellis structure on performance of concatenation scheme is shown in Fig.3, in which (a) is BER performance curves without interleaver and (b) is BER performance curves with interleaver. The X-axis in the figure means signal noise ratio and the y-axis means bit error rate. From the curves shown in the Fig.3, we can get several conclusions as follows: 1) non-recursive trellis FQPSK outperforms recursive one whether with interleaver or not, which is reverse to that when FQPSK is concatenated with convolutional code, 2) FQPSK with non- recursive trellis outperforms recursive one more than $0.6 \mathrm{~dB}$ when a interleaver is used, and the improvement is up to $1.6 \mathrm{~dB}$ while without interleaver, 3) CEFQPSK performs better than FQPSK about $0.2 \mathrm{~dB}$ no matter under which case.

From the Fig.3, we can see interleaver has a noticeable impact on performance of iterative receiving. The effect has been illustrated in detail in subsequent parts by simulation. In the simulation, the iteration times set 10, BER performance are compared between adopting an interleaver in system or not. The result is shown in Fig.4, in which (a) is BER performance curves of FQPSK with recursive trellis and (b) is BER performance curves of it with non-recursive trellis. From the curves shown in the Fig.4, we can get several conclusions as follows. 1) There is about $1 \mathrm{~dB}$ improvement for recursive FQPSK (CEEFQPSK) that resulted from interleaver. 2) For non-recursive FQPSK, interleaver will not work better any.

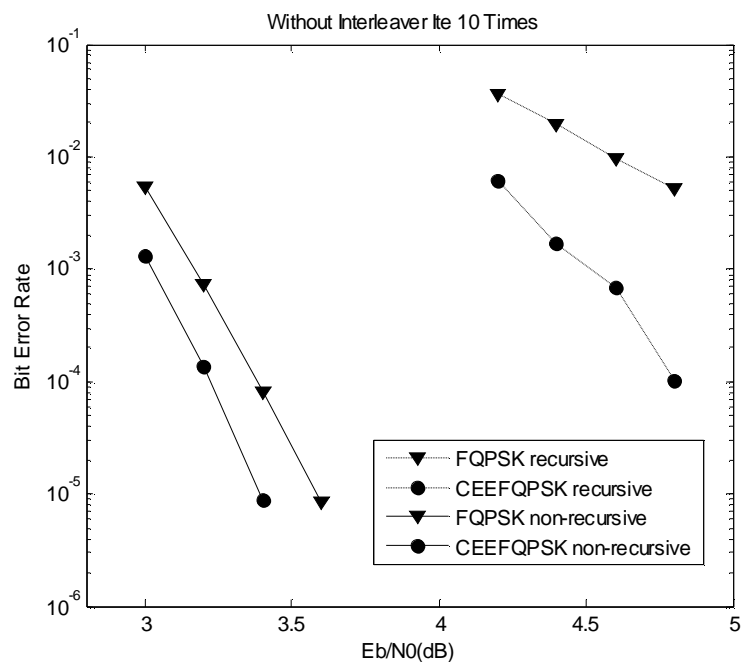

(a)

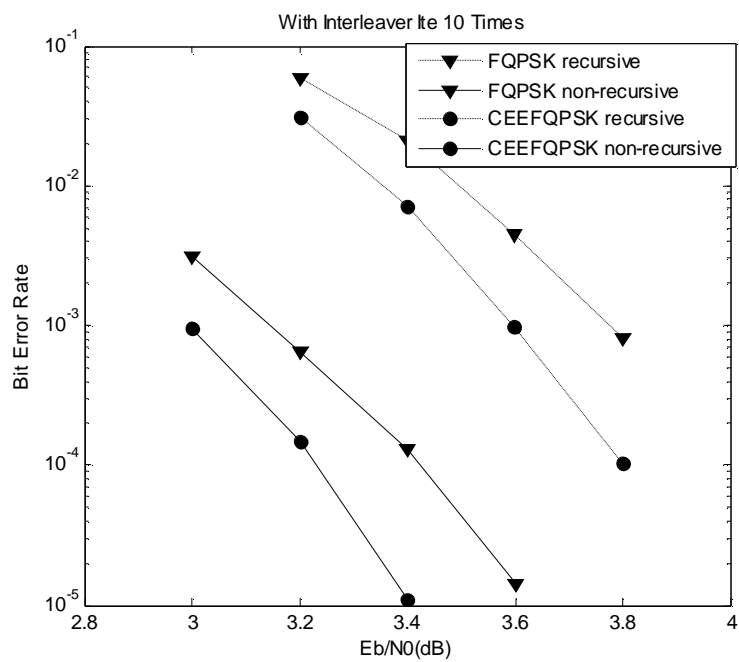

(b)

Fig.3 BER performance vs. trellis structure of FQPSK with iterative demodulation (a) without interleaver, (b) with interleaver 


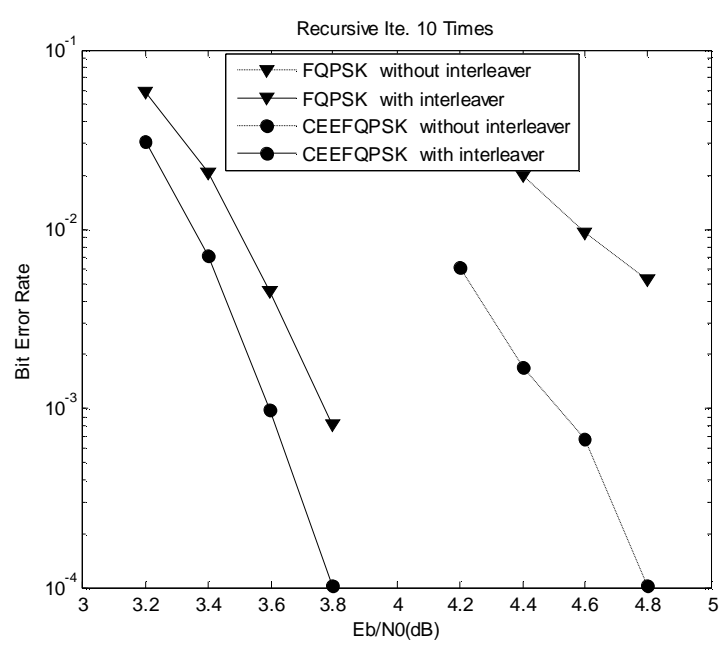

(a)

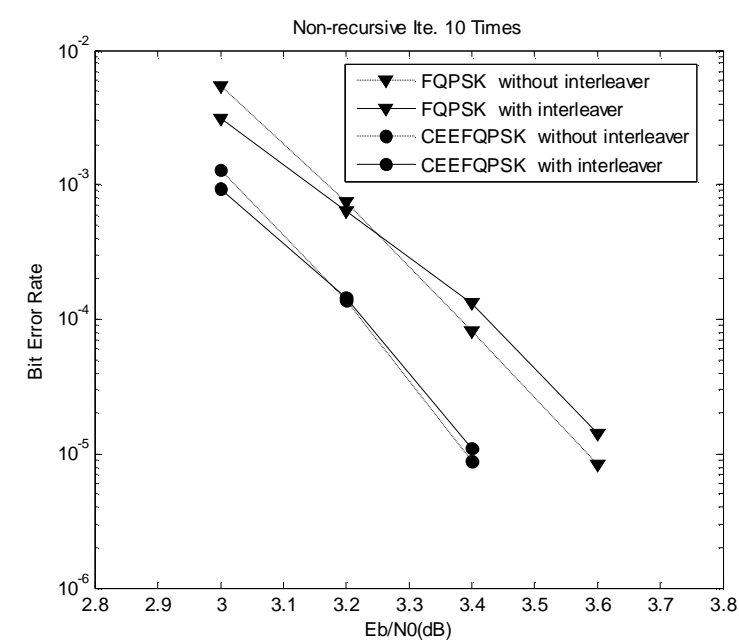

(b)

Fig.4 BER performance vs. interleaver of FQPSK with iterative demodulation (a) recursive (b) nonrecursive

\section{BER Performance of Optimized Scheme.}

From above optimization results, the concatenation scheme that non-recursive CEEFQPSK without interleaver performs better than others. Fig. 5 shows the relation between optimized scheme BER performance and iteration times. The $\mathrm{x}$-axis in the figure means iteration times and the $\mathrm{y}$-axis means bit error rate. From the figure, we know that BER performance can be improved drastically when iteration up to 10 times and it will be lower than $10^{-5}$ when signal noise ratio is $3.4 \mathrm{~dB}$ for the optimized scheme, while signal noise ratio is $3.0 \mathrm{~dB}$ the iteration times is 13 for BER down to $10^{-5}$.

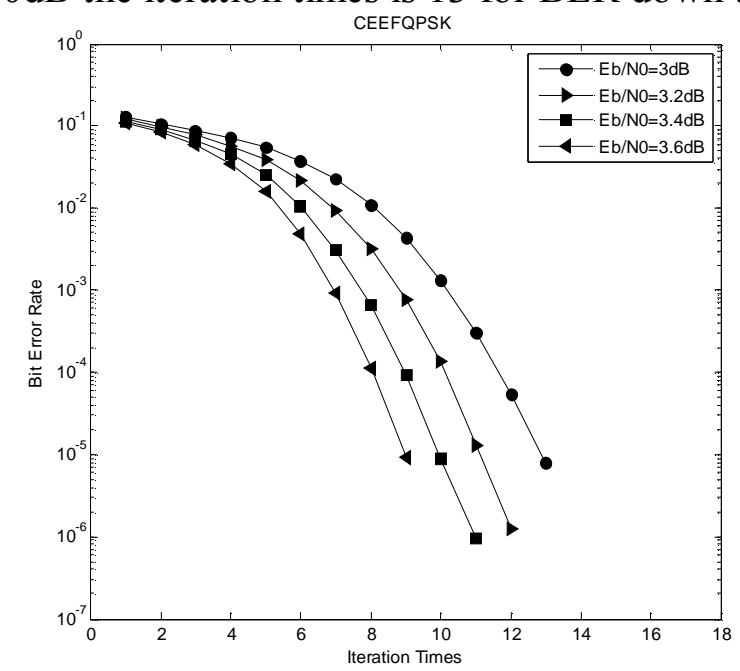

Fig.5 BER performance of optimized scheme

\section{Conclusions}

Motivated by the potentially large gain achievable with iterative receiving, one novel coded modulation scheme based on LDPC code and FQPSK (Feher's QPSK) is designed and optimized in this paper, which can alleviate the challenge of signal power huge loss in space communications. Due to the fact that the FQPSK modulation and AR4JA code both are recommendatory by CCSDS, it is one of most promising concatenation scheme in power limited space communications system. 


\section{Acknowledgment}

The authors would like to thank the Project Sponsored by National Natural Science Foundation of China (61401507, 91438109, 91338201) and“863" Program of China (2012AA01A510)

\section{References}

[1] Z. J. Yu, "Deep space telecommunication system,", Beijing: National Defence Industry Press, pp:10-20, 2009

[2] K. S. Andrews, D. Divsalar, "The development of Turbo and LDPC codes for deep space applications," Proceedings of the IEEE, Vol.95, No.11, pp: 2142-2156, 2007.

[3] CCSDS 131.1-O-2 "Low density parity check codes for use in near-earth and deep space applications," CCSDS, 2007.

[4] M. K. Simon, "Bandwidth efficient digital modulation with application to deep space communications," New York: Wiley, pp.125-166, 2003.

[5] K. Feher, "FQPSK: a superior modulation technique for mobile and personal communications," IEEE Trans. on Broadcasting, Vol.39, No.2, pp: 288-294, 1993.

[6] Z. D. Xie, G. X. Zhang. D.M. Bian, "Constant envelope enhanced FQPSK and its performance analysis," Journal of Communications and Network, Vol.13, No.5, pp: 442-448, 2011.

[7] S. Benedetto, D. Divsalar, G. Montorsi, F. Pollara, "A Soft-Input Soft-Output APP module for iterative decoding of concatenated codes," IEEE Communications Letters, Vol.1, No.1, pp: 22-24, 1997.

[8] M. Franceschini, G. Ferrari, R. Raheli, and A. Curtoni, "Serial concatenation of LDPC codes and differential modulations," IEEE Journal On Selected Areas In Communications, Vol.23, No.9, pp: 1758-1768, 2005.

[9] S. T. Brink, G. Kramer, and A. Ashikhmin, "Design of Low-Density Parity-Check codes for modulation and detection," IEEE Transactions On Communications, Vol.52, No.4, pp: 670-678, 2004.

[10]J. R. Bao, Y. F. Zhan, J. H. Lu, "High efficiently joint LDPC coded recursive MSK modulation," Journal of Tsinghua University, Vol.50, No.1, pp: 104-107, 2010.

[11] W. Guan, M. K. Dong, H. G. Xiang, "A joint demodulation and decoding algorithm for high order LDPC coded modulations," Journal of Electronics \& Information Technology, Vol.32, No.2, pp: 340-344 2010.

[12]L. F. Li, M. K. Simon, "Performance of coded OQPSK and MIL-STD SOQPSK with iterative decoding," IEEE Transactions On Communications, Vol.52, No.11, pp: 1890-1900, 2004.

[13]E. Perrins, T. Nelson, M. Rice. "Coded FQPSK and SOQPSK with iterative detection," Military Communications Conference, 2005.

[14] G. X. Zhang, D. M. Bian, J. Hu, Z. D. Xie, "A concatenated coded modulation scheme and its iterative receiving for deep space communications," ChinaCom, Haerbin, August,2011. 